\title{
Response to Comment on "Log or Linear? Distinct Intuitions of the Number Scale in Western and Amazonian Indigene Cultures"
}

\author{
Stanislas Dehaene ${ }^{1,2,3,4,{ }^{*}, \text { Véronique Izard }}{ }^{1,2,4,5}$, Pierre Pica ${ }^{6}$, and Elizabeth Spelke ${ }^{5}$ \\ ${ }^{1}$ Institut National de la Santé et de la Recherche Médicale (INSERM), Cognitive Neuroimaging \\ Unit, Institut Fédératif de Recherche (IFR) 49, Gif-sur-Yvette, France. \\ ${ }^{2}$ Commissariat àl'Energie Atomique, NeuroSpin Center, IFR 49, Gif-sur-Yvette, France. \\ ${ }^{3}$ Collège de France, Paris, France. \\ 4Université Paris-Sud, IFR49, F-91191 Gif-sur-Yvette, France. \\ ${ }^{5}$ Department of Psychology, Harvard University, Cambridge, MA 02139, USA. \\ 'Unité Mixte de Recherche 7023 "Formal Structure of Language," CNRS and Paris VIII \\ University, Paris, France.
}

\begin{abstract}
The performance of the Mundurucu on the number-space task may exemplify a general competence for drawing analogies between space and other linear dimensions, but Mundurucu participants spontaneously chose number when other dimensions were available. Response placement may not reflect the subjective scale for numbers, but Cantlon et al.'s proposal of a linear scale with scalar variability requires additional hypotheses that are problematic.
\end{abstract}

\begin{abstract}
Our study (1) investigated how the Mundurucu, an Amazonian indigenous people with little or no formal education, map numbers onto space. We agree with Cantlon et al. (2) that the performance of the Mundurucu on our number-line task could exemplify a more general capacity for analogical reasoning that allows mappings between space and other linear dimensions. The mapping of number to space is surely not the only mapping available to the human mind; indeed, one of our earlier studies provided evidence that the Mundurucu spontaneously relate large-scale three-dimensional spatial layouts to small two-dimensional geometric forms, using the latter as literal maps (3). Humans may well possess a generic capacity to think of all quantities, be they distances, object sizes, or any other continuous dimension, as fundamentally commensurate and assessable by a single measurement system (real numbers). Nevertheless, the appeal to analogy raises the crucial question of whether some stimulus dimensions are privileged when mapping stimuli onto space. In our study (1), the target sets varied on multiple dimensions, including element size, brightness, average area, and number, and each of these dimensions could have been mapped onto space. The two training trials provided insufficient instruction or feedback to fully distinguish between these possible mappings. Still, the Mundurucus spontaneously selected number as the main dimension underlying their pointing responses. This systematic pattern provides evidence that the mapping of number to space is intuitive and privileged.
\end{abstract}

Is the subjective scale of number logarithmic or linear? On this issue, Cantlon et al. (2) propose an alternative interpretation of our findings. Although the number-line responses of

\footnotetext{
Copyright 2009 by the American Association for the Advancement of Science; all rights reserved.

*To whom correspondence should be addressed. stanislas.dehaene@cea.fr.
} 
the Mundurucu are logarithmically spaced, their internal representation of number would be linear, with equal psychological distance between adjacent numbers but with linearly increasing variability. Cantlon et al's suggestion runs counter to the simplest construal of number-line placements as indicators of psychological distance. On this standard construal $(4,5)$, participants evaluate the size of the numbers and place them at spatial distances relative to the endpoints that are proportional to their psychological distances from those endpoints. The challenge for the hypothesis of a linear code with scalar variability is to formulate what it would mean to possess a linear psychological distance metric and yet respond logarithmically in a test as simple as ours.

Cantlon et al. rise to this challenge by supposing that the number-line task is not as simple as it seems but involves "a ratio comparison process between the anchors and the probe values." This means that participants do not report the psychological distance between each probe number and the anchors (i.e., their difference, which would be linear) but the similarity between them (which takes into account their internal variability and hence varies with their ratio). In brief, on this account, the Mundurucu would base their spatial responses on perceived number similarity, all the while possessing a linear sense of psychological distance.

This account, however, is problematic for several reasons. First, it depends on the assumption that perceived similarity can be finely evaluated quantitatively, not only at threshold but also well above threshold (since quantities such as 3 are easily discriminable from both 1 and 10, even for infants). Second, this account posits two internal metrics, one of similarity and a distinct one of psychological distance, with the assumption that, despite what the term "distance" implies, the second one is not easily mapped onto space. Third, this account provides no explanation for the changes in task performance that occur with intercultural contact or education, without additional assumptions that are either highly implausible or demonstrably false. In our experiments, Mundurucu participants who could count in Portuguese showed a linear response with Portuguese number words but a logarithmic response with dot stimuli and with Mundurucu number words. In other experiments using this task $(4,5)$, young children in U.S. elementary schools showed linear performance with a number line scaled from 1 to 100 but logarithmic performance at larger scales. To account for these performance patterns, Cantlon et al. (2) might propose that bilingual Mundurucu adults and U.S. school children learned to construe the number-line task differently. However, if these participants somehow learned that the task required mapping of psychological distance when the stimuli were Portuguese words or small numbers, and if they were endowed with a linear sense of numerical distances, then why did they fail to apply this mapping more broadly? Alternatively, Cantlon et al. could propose that the similarity relations among numbers change during development, as the linear code with scalar variability is replaced by a linear code with fixed variability. Although such a developmental change may occur, a large amount of data from numerosity discrimination (6), nonverbal arithmetic $(7,8)$, magnitude estimation (9), and subjective similarity reports of symbolic numerals (10) shows that even in educated adults, number similarity still varies with numerical ratio or, equivalently, logarithmic distance. We therefore stand by the original hypothesis $(1,4,5)$ : Young children begin with a logarithmic sense of number, and education subsequently provides an additional linear representation, suitable for mapping numbers onto space, but which does not totally supplant the logarithmic representation in all tasks $(1,11,12)$.

Since Fechner (13), Stevens (14), and Krueger (15), the issue of mapping from objective to subjective quantity has become increasingly technical. Cantlon et al. correctly point out that the logarithmic code and the linear code with scalar variability often make identical behavioral predictions, because both predict ratio-based numerical discrimination. The two 
models are not, however, empirically indistinguishable. A subtle but distinctive prediction concerns the shape of the internal noise: According to the logarithmic hypothesis, it should be Gaussian on a log scale, and therefore the distribution should be rightward skewed when plotted on a linear scale. Conversely, in some carefully designed situations (departing from mere discrimination), the linear model predicts a Gaussian distribution of responses on a linear scale, and therefore a leftward skewed distribution on a log scale. Several studies have attempted to characterize the noise distribution for number, either behaviorally (12) or, most crucially, with methods that directly probe the neural code for numerosity in monkeys and humans $(16,17)$. All results so far support the logarithmic model.

\section{Acknowledgments}

Supported by INSERM, the Départment des Sciences Humaines et Sociales of CNRS (P.P.), NIH (E.S.), and a McDonnell Foundation centennial fellowship (S.D.).

\section{References and Notes}

1. Dehaene S, Izard V, Spelke E, Pica P. Science. 2008; 320:1217. [PubMed: 18511690]

2. Cantlon JF, Cordes S, Libertus ME, Brannon EM. Science. 2009; 323:5910. www.sciencemag.org/ cgi/content/full/323/5910/38b.

3. Dehaene S, Izard V, Pica P, Spelke E. Science. 2006; 311:381. [PubMed: 16424341]

4. Siegler RS, Opfer JE. Psychol. Sci. 2003; 14:237. [PubMed: 12741747]

5. Siegler RS, Booth JL. Child Dev. 2004; 75:428. [PubMed: 15056197]

6. van Oeffelen MP, Vos PG. Percept. Psychophys. 1982; 32:163. [PubMed: 7145586]

7. Cordes S, Gelman R, Gallistel CR, Whalen J. Psychon. Bull. Rev. 2001; 8:698. [PubMed: 11848588]

8. Barth H, et al. Cognition. 2006; 98:199. [PubMed: 15876429]

9. Izard V, Dehaene S. Cognition. 2008; 106:1221. [PubMed: 17678639]

10. Shepard RN, Kilpatrick DW, Cunningham JP. Cognit. Psychol. 1975; 7:82.

11. Banks WP, Coleman MJ. Percept. Psychophys. 1981; 29:95. [PubMed: 7255104]

12. Dehaene S, Marques JF. Q. J. Exp. Psychol. 2002; 55:705.

13. Fechner, GT. Elemente der Psychophysik. Breitkopf and Hartel; Leipzig: 1860.

14. Stevens SS. Psych. Rev. 1957; 64:153.

15. Krueger LE. Behav. Brain Sci. 1989; 12:251.

16. Nieder A, Miller EK. Neuron. 2003; 37:149. [PubMed: 12526780]

17. Piazza M, Izard V, Pinel P, Le Bihan D, Dehaene S. Neuron. 2004; 44:547. [PubMed: 15504333] 